\title{
Novel bi-allelic variants expand the SPTBN4-related genetic and phenotypic spectrum
}

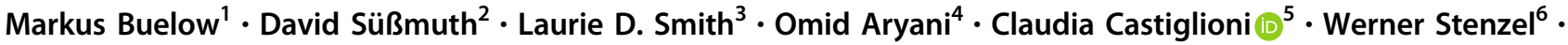 \\ Enrico Bertini ${ }^{7} \cdot$ Markus Schuelke $\mathbb{1}^{1,8} \cdot$ Ellen Knierim $\mathbb{1}^{1,8}$
}

Received: 23 July 2020 / Revised: 4 February 2021 / Accepted: 25 February 2021 / Published online: 26 March 2021

(c) The Author(s) 2021. This article is published with open access

\begin{abstract}
Neurodevelopmental disorder with hypotonia, neuropathy, and deafness (NEDHND, OMIM \#617519) is an autosomal recessive disease caused by homozygous or compound heterozygous variants in SPTBN 4 coding for type $4 \beta I V$-spectrin, a non-erythrocytic member of the $\beta$-spectrin family. Variants in SPTBN4 disrupt the cytoskeletal machinery that controls proper localization of ion channels and the function of axonal domains, thereby generating severe neurological dysfunction. We set out to analyze the genetic causes and describe the clinical spectrum of suspected cases of NEDHND. Variant screening was done by whole exome sequencing; clinical phenotypes were described according to the human phenotype ontology, and histochemical analysis was performed with disease-specific antibodies. We report four families with five patients harboring novel homozygous and compound heterozygous SPTBN4 variants, amongst them a multi-exon deletion of SPTBN4. All patients presented with the key features of NEDHND; severe muscular hypotonia, dysphagia, absent speech, gross motor, and mental retardation. Additional symptoms comprised horizontal nystagmus, epileptiform discharges in EEG without manifest seizures, and choreoathetosis. Muscle histology revealed both characteristics of myopathy and of neuropathy. This report expands the SPTBN4 variant spectrum, highlights the spectrum of morphological phenotypes of NEDHND-patients, and reveals clinical similarities between the NEDHND, non-5q SMA, and congenital myopathies.
\end{abstract}

Ellen Knierim

ellen.knierim@charite.de

1 Department of Neuropediatrics, Charité - Universitätsmedizin Berlin, corporate member of Freie Universität Berlin und Humboldt Universität zu Berlin, Berlin, Germany

2 HELIOS Kliniken - Helios Klinikum Hohenstücken, Berlin, Germany

3 Department of Pediatrics, Division of Pediatric Genetics and Metabolism, The University of North Carolina SOM, North Carolina, NC, USA

4 Department of Neuroscience, Iranian University of Medical Sciences, Tehran, Iran

5 Pediatric Neurology, Clínica Las Condes, Santiago, Chile

6 Department of Neuropathology, Charité - Universitätsmedizin Berlin, corporate member of Freie Universität Berlin und Humboldt Universität zu Berlin, Berlin, Germany

7 Unit of Neuromuscular and Neurodegenerative Disorders, Bambino Gesù Children's Research Hospital, IRCCS, Rome, Italy

8 NeuroCure Clinical Research Center, Charité -

Universitätsmedizin Berlin, corporate member of Freie Universität Berlin und Humboldt Universität zu Berlin, Berlin, Germany

\section{Introduction}

Spectrins are cytoskeletal proteins found in a variety of tissues and cell types. They were initially identified in erythrocytes [1]. Vertebrate spectrin is a hetero-tetramer formed by two $\alpha$ - and two $\beta$-subunits. The $\alpha$-spectrin subgroup has two members (I and II), while the $\beta$-spectrin subgroup has five members (I-V). $\beta \mathrm{IV}$-spectrin is enriched in the myelinated neurons of the central nervous system [2], where it has a role in the clustering of sodium and potassium channels at the axon initial segment (AIS) and at the nodes of Ranvier via interaction with ankyrin-G [3]. The $S P T B N 4$ gene encodes a non-erythrocytic $\beta I V$-spectrin. We published the first case of autosomal recessive myopathy caused by a homozygous pathogenic SPTBN4 variant in 2017 [4]. The proband was boy of Kurdish descent who, in addition to the classical symptoms of myopathy, exhibited sensorineural hearing loss, intellectual disability, and Spectrin-associated neuropathy. A recent report by Wang et al. [5] described six additional children with this disease due to autosomal recessive SPTBN4 variants. They demonstrated that a loss-of-function variant disrupted 
sodium and potassium channel clustering, leading to neuropathy. Häusler et al. reported a novel homozygous splicesite variant in a pair of siblings presenting with axonal neuropathy in the absence of intellectual disability [6]. In pigs, deletions in SPTBN4 cause severe myopathy [7]. We now report five additional affected individuals from four families who were found to harbor variants that affect function of SPTBN4. Our findings further define the clinical spectrum of neurodevelopmental disorder with hypotonia, neuropathy, and deafness.

\section{Materials and methods}

\section{Patients}

The patients' parents provided written informed consent for study participation, including publication of patient photographs, in accordance with the Declaration of Helsinki (Charité Ethics Committee approval EA2/107/14). Patients 1 and 2 were admitted to the Helios Klinikum Hohenstücken in Berlin, Germany, for neuro-rehabilitation; Patient 3 presented as an outpatient at UNC Chapel Hill's Division of Pediatric Genetics and Metabolism, Chapel Hill, NC, USA; Patient 4 presented as an outpatient at the Iranian University of Medical Sciences, Tehran, Iran; and Patient 5 was treated and diagnosed at the Clínica Las Condes, Pediatric Neurology, Santiago, Chile.

\section{Sequencing and segregation analysis}

Genomic DNA was extracted from white blood cells and whole exome sequencing (WES) was performed for all patients. Variant interpretation was performed according to current ACMG guidelines for variant classification [8]. We submitted our variants to ClinVar (VCV000987745, VCV0009877446, VCV000987747, VCV000988586).

\section{Histology, immunohistochemistry (IHC), and morphometry}

Fiber-type-specific atrophy was measured by determining the minimal Feret's diameter of each fiber from muscle cross-sections using a standardized method. The use of the minimal Feret's diameter minimizes the confounding factor of oblique sectioning. For calculating the minimal Feret's diameter all fibers of one representative $200 \times$ field of view of a ATPase $\mathrm{pH} 4.3$ stained muscle section were counted. This amounted to $n=131$ type 1 and $n=156$ type 2 fibers in the patient, and $n=177$ type 1 and $n=174$ type 2 fibers in the control. IHC staining and imaging was performed as previously described [4]. Images were loaded into ImageJ (https://imagej.nih.gov/ij/) processing software, the circumference of individual fibers was manually traced and the minimal Feret's diameter calculated using the build-in measuring algorithm. Values were visualized as cumulative histograms in GraphPad Prism (GraphPad Software Inc., San Diego, CA, USA).

\section{Results}

\section{Clinical reports}

\section{Family A (Patients 1 and 2)}

Two affected sisters were born at term to healthy first-cousin parents from Saudi Arabia. The pregnancy with Patient 1 was complicated by gestational diabetes. Muscular hypotonia was noted at birth. Feeding problems became apparent during the newborn period. She suffered from recurrent aspiration pneumonia and dysphagia from the first month of life, requiring gavage feeding from 1 year of age. Motor development was severely delayed, and the patient did not achieve early developmental milestones such as head control, rolling over, and crawling (Table 1). A physical examination at age 3 years revealed a high palate, myopathic facies, severe distal muscle weakness, generalized amyotrophy, bilateral ankle flexion contractures (Fig. 1A, B), and severe global developmental delay. Patient 1 was unable to stand, sit, eat, or drink without support at age 3 years. Her speech was limited to repeating single words in Arabic and German. Neurometabolic screening tests (tandem mass spectrometry of amino and organic acids, lactate, ammonia) and first-line genetic analyses (karyotyping, muscular dystrophy gene panel) were inconclusive. Serum CPK levels were normal. Cranial MRI revealed a diffuse $\mathrm{T}_{2}$-hyperintensity, predominantly affecting the subcortical white matter. Structural abnormalities were ruled out. MR spectroscopy was normal. A muscle biopsy was not performed. The younger sister of the patient (Patient 2) presented in a similar way but was more severely affected at the age of 2 years.

\section{Family B (Patient 3)}

Patient 3 is a 5-year-old female and the first child born to healthy paternal second cousins from Afghanistan. Pregnancy and birth were normal. Muscle hypotonia was diagnosed in the neonatal period. She suffered from recurrent pulmonary aspiration starting at 5-6 months of age. She had developed neither head control, independent sitting, crawling, nor talking by 2 years of age. A physical exam revealed horizontal nystagmus, choreoathetosis of the arms with intermittent dystonia, and generalized hypotonia (Fig. 1D). Deep tendon reflexes were brisk without clonus. Laboratory testing for inborn errors of metabolism, routine karyotyping, and 


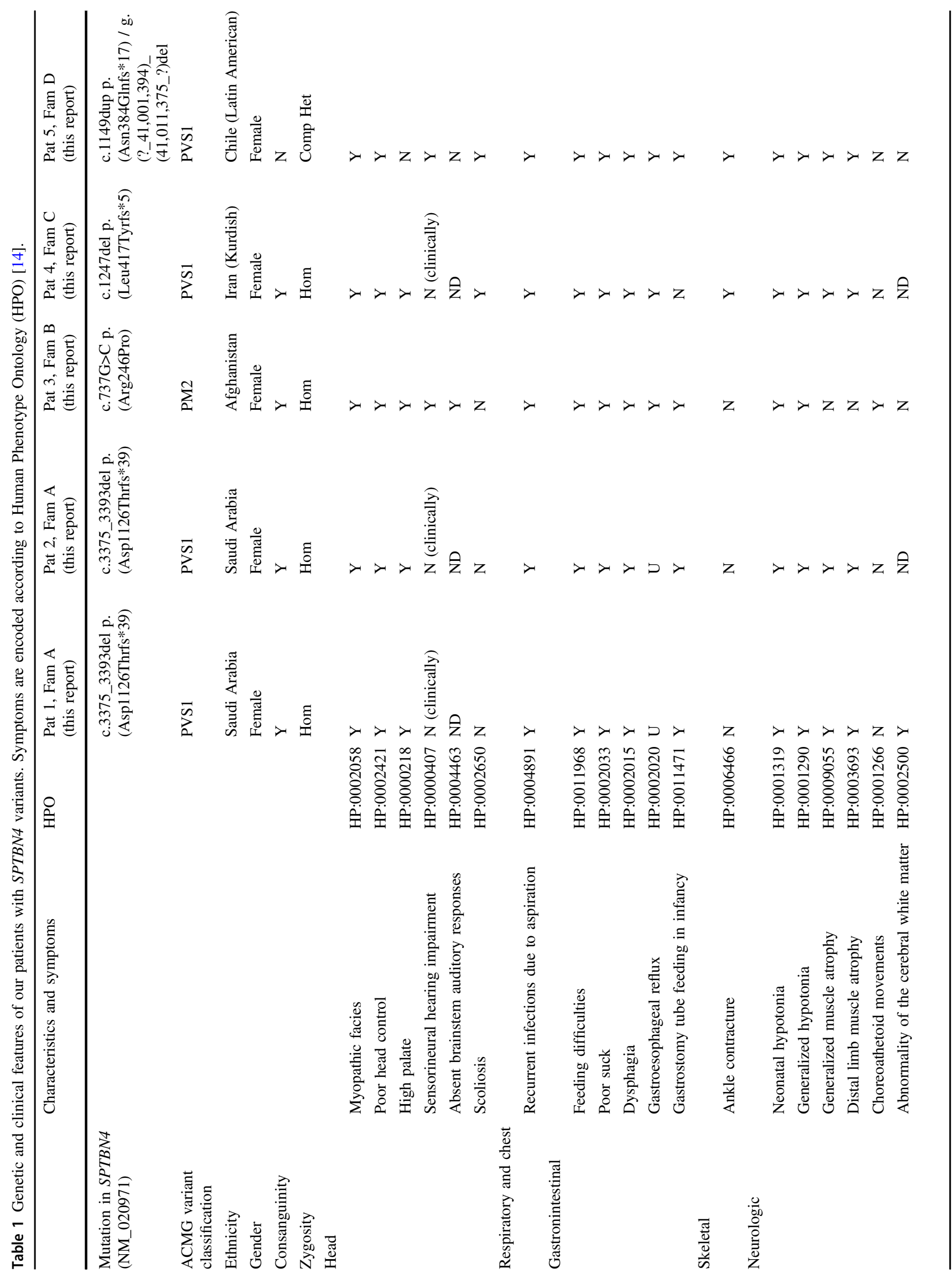




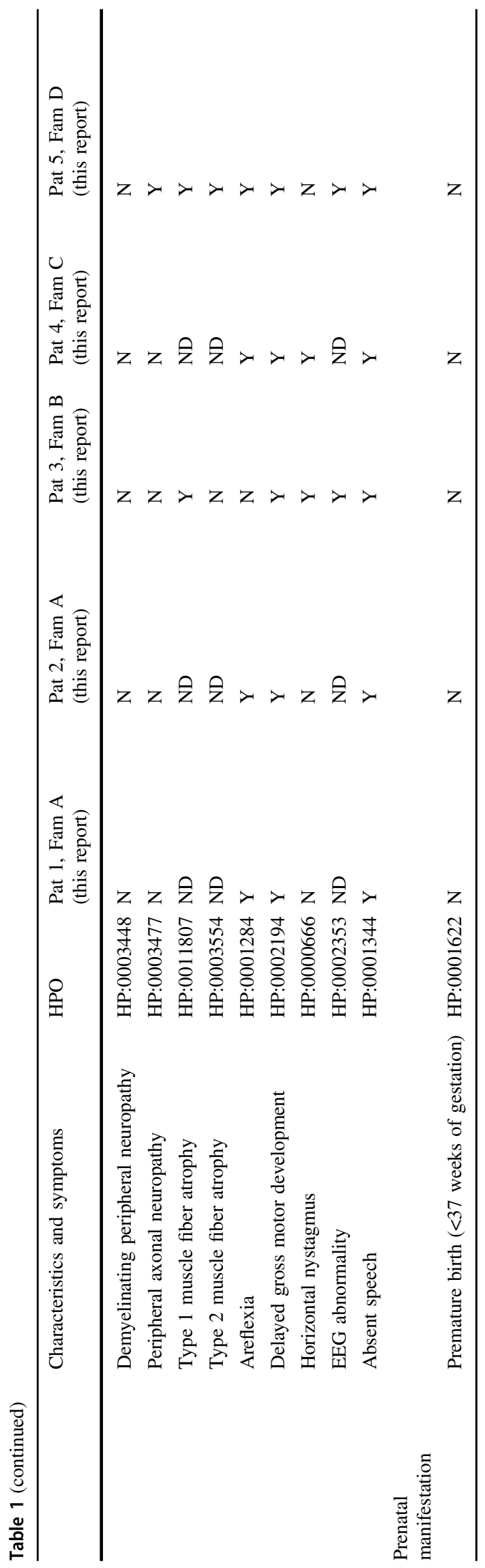

microarray analysis were inconclusive. Cranial MRI and CT were normal. MRS revealed nonspecific lipid and lactate peaks with increased glutamine/glutamate peaks in the region of the basal ganglia and the Corpus callosum. Oral feeding led to recurrent aspiration pneumonias and dystrophy with a body weight below the first percentile. Gastrostomy feeding was started at 3 years of age. Seventy-two-hour EEG showed abundant to nearly continuous centro-parietal sharp/spike/ poly-spike wave discharges during sleep without clinical correlation to manifest seizures. Nerve conduction studies and EMG were entirely normal. Otoacoustic emissions were present. In brainstem evoked response audiometry (BERA) only wave 1 from the inner ear could be recorded pointing to a defect at the level of cochlear nerve conduction.

\section{Family C (Patient 4)}

Patient 4 is a 4-year-old female born to healthy consanguineous parents from Iran with Kurdish background. Her older brother died at age 2 years due to increasing feeding difficulties and subsequent aspiration pneumonia. His phenotype was described as similar. Patient 4 showed signs of general hypotonia and muscle weakness shortly after birth (Fig. 1F). Routine metabolic testing, including acylcarnitine and urine organic acids, was normal. Genetic testing for spinal muscular atrophy type 1 was negative. She had poor head control and neither sat, crawled, nor spoke, and had severe dystrophy (Fig. 1G). She presented with clinical signs of myopathy, including myopathic facies, high arched palate, and bilateral ankle flexion contractures. She had horizontal nystagmus. EEG or BERA were not recorded. Sensory-nerve action potentials and motor action potentials were normal.

\section{Family D (Patient 5)}

Patient 5 is a 7-year-old girl born to healthy nonconsanguineous parents from Chile. She was born at term, following a normal pregnancy and delivery. Her parents noted a weak suck and slow weight gain during her first week of life. Muscular hypotonia, motor delay, and frequent choking and gagging while feeding were noted at 3 months of age. Severe gastroesophageal reflux with nasal regurgitation led to recurrent respiratory infections. Patient 5 had attained only partial head control and could neither sit nor stand (Fig. 1I, J). An examination at 10 months of age showed mild weakness of facial musculature, severe hypotonia without trunk control, bilateral Talipes equinovarus and Pes cavus, and absent deep tendon reflexes. Though able to exert spontaneous antigravity movements of the upper and lower limbs, she was unable to lift her head when prone. Bilateral moderate to severe hearing loss was diagnosed at 18 months of age, at 3 years her speech recognition threshold was $80 \mathrm{~dB}$ HL. Her growth parameters at age 4 


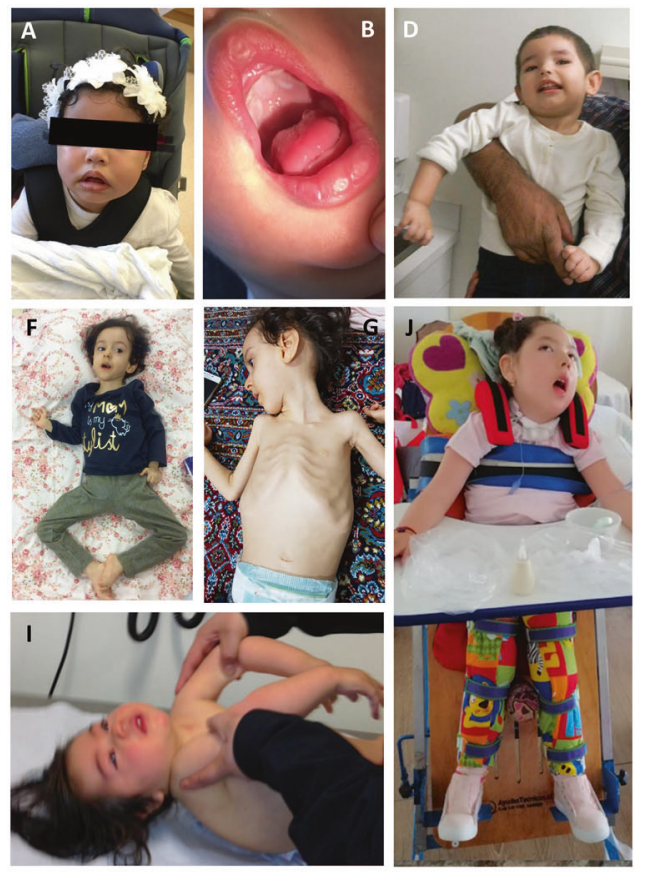

Fig. 1 Clinical images of the patients and family trees. A Patient 2 (II.2) from Family A at age 2.5 years. B Note the tented upper lip vermillion, her mask-like facies, and the high palate. C Pedigree of Family A. D Patient 3 (II.2) from Family B at age 5. Note the lack of head control, choreoathetoid arm movements, and muscular hypotonia causing slip through when held in vertical position under the armpits. E Pedigree of Family B. F, G Patient 4 (II.2) from Family C at age 4 years. Note severe dystrophy due to feeding difficulties and unavailability of gastric tube feeding, myopathic facies, and severe muscle

years were below the third percentile. Tracheostomy and gastrostomy were required due to increasing dysphagia and a weak cough reflex coupled with recurrent pneumonias. Karyotyping, serum CPK levels, Prader-Willi syndrome (tested by methylation analysis), and spinal muscular atrophy type 1 genetic testing were normal. cMRI did not show any abnormalities. Nerve conduction studies showed normal sensory and motor conduction velocities, with lowamplitude motor responses. EMG showed signs of acute and chronic denervation such as fibrillation potentials and positive sharp waves, motor unit potentials with increased amplitude and duration, and decreased recruitment. An EEG at age 6 years revealed almost continuous and severe generalized epileptiform activity without any clinical correlate of manifest seizures (Fig. 1L).

\section{Whole exome sequencing identifies variants in SPTBN4}

WES of the patients and their parents revealed novel bi-allelic variants in SPTBN4 in all four families. Deletions were found in Families A and C. Patient 1 and 2 from Family A were homozygous for

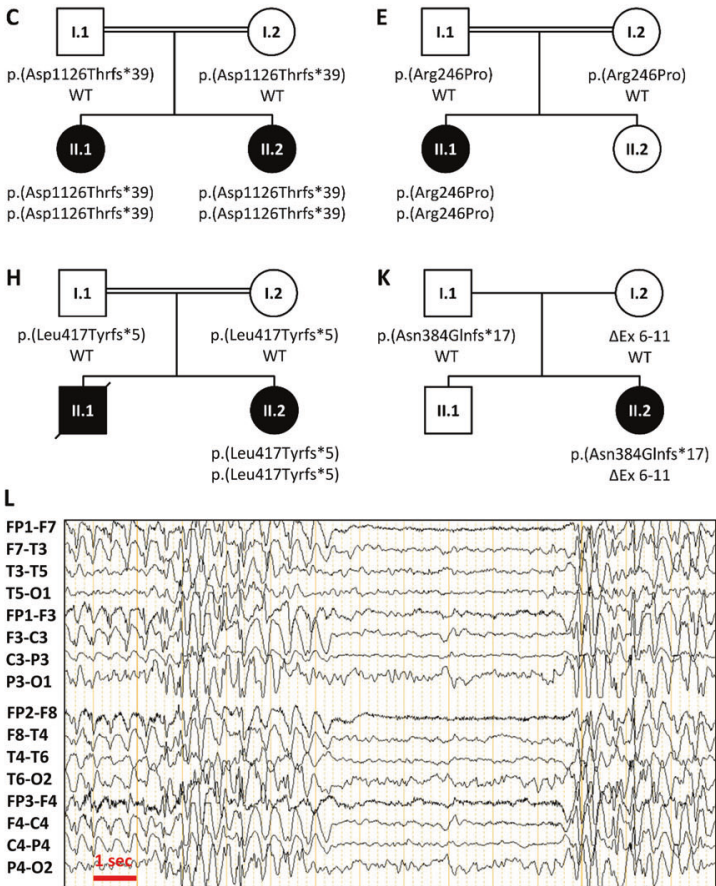

weakness with frog-leg posture. Her brother (II.1) passed away from aspiration pneumonia and had a similar phenotype. H Pedigree of Family C. I, J Patient 5 from Family D, I shows the severe muscle hypotonia, $\mathbf{J}$ depicts the same patient at 6 years not able to stand independently. K Pedigree of Family D. L EEG from Patient 5 at age 6 years shows generalized epileptic activity. No clinical seizures were observed in a 24-h video EEG recording. The parents had never observed any type of fits or seizures.

41,025,797del (hg19); NM_020971.3c.3375_3393del; p. (Asp1126Thrfs*39)] (Fig. 1C) and Patient 4 from Family C was homozygous for [chr19:g.41,008,725_41,008,725del (GRCh37); NM_020971.3c.1247del; p.(Leu417Tyrfs*5)]. These deletions cause frameshifts that lead to a premature stop codon. Sanger sequencing was done in Family A and verified the variant and its segregation. Both parents were heterozygous. Patient 3 from Family B was homozygous for the missense variant [chr19:g.41,003,464 (GRCh37); NM_020971.3c.737G >C; p.(Arg246Pro)] (Fig. 1E) that was absent from gnomAD. Patient 5 from Family D was compound heterozygous for two variants. The paternally inherited single base insertion [chr19:g.41,008,360dup (GRCh37); NM_020971.3c.1149dup; p.(Asn384Glnfs*17)] causes a frameshift that leads to a subsequent premature stop codon. The maternally inherited deletion with breakpoint spanning [chr19.g.(?_41,001,394)_(41,011,375_?)del

(GRCh37)] encompasses exons $6-11$ of SPTBN4 (Fig. 1K).

\section{Histology and morphometry}

Muscle biopsy was performed in Patients 3 and 5. In Patient 3, ATPase $\mathrm{pH} 4.3$ staining and IHC with an antibody 

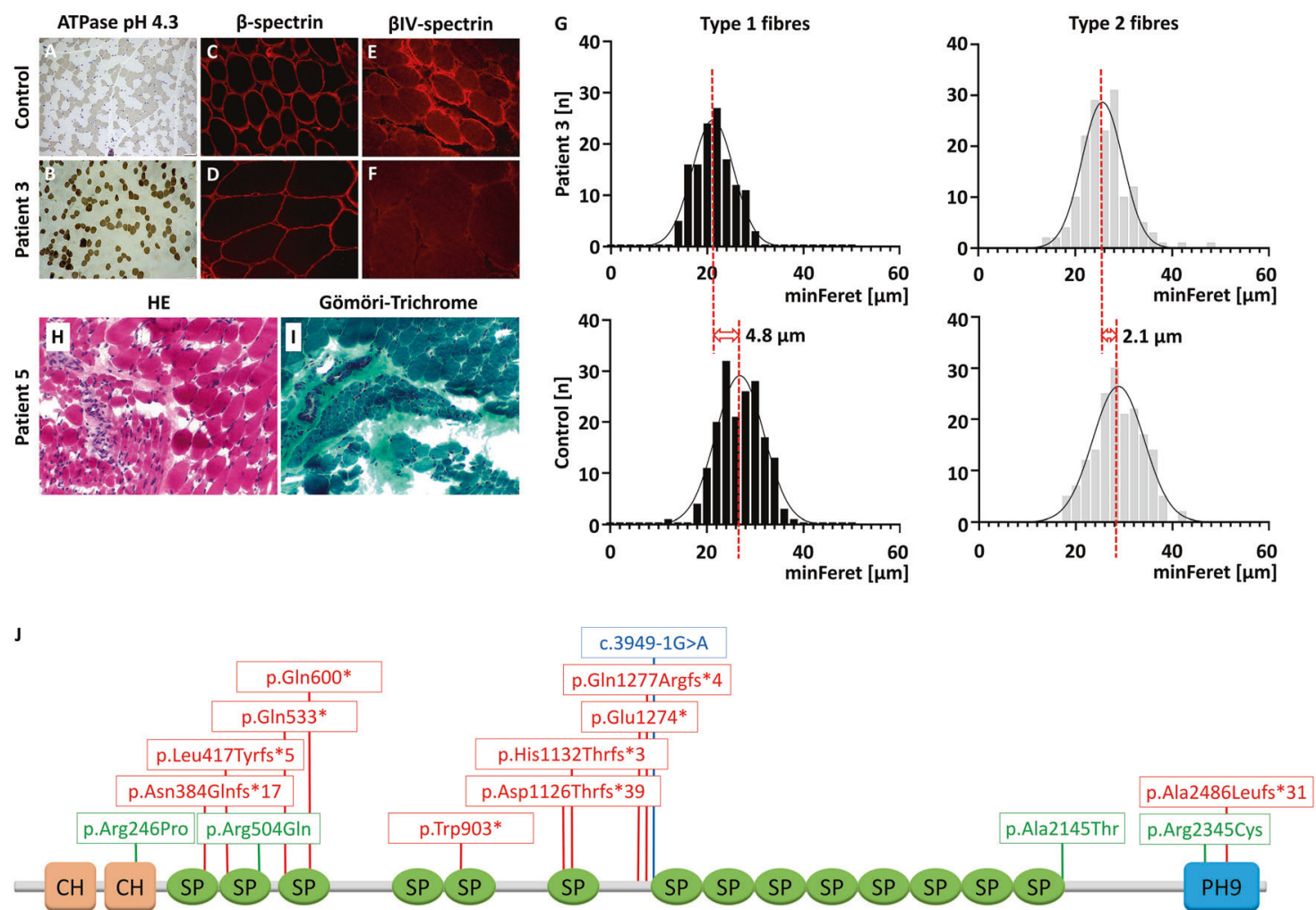

$\mathrm{CH}$ Calponin homology domain

SP Spectrin repeat

PH9 Pleckstrin homology domain

Fig. 2 Histological and morphometric investigations. A, B ATPase pH 4.3 staining showing type 1 fiber (dark) hypotrophy in Patient 3 (B) in comparison to an age-matched control (A) (200x, scale bar 50 $\mu \mathrm{m})$. Immunostaining of the same samples with an antibody directed against $\beta$-spectrin showed staining of the sarcolemma in both individuals $(\mathbf{C}, \mathbf{D}, 600 \times$ and $200 \times$, respectively). Staining for $\beta$ IV-spectrin did not show any signal at the sarcolemmal position of the Patient 3 (F) in contrast to a healthy control (E) (400x). G Histogram of diameters from type 1 and type 2 muscle fibers from Patient 3 and an age-matched control showing an over-proportional thinning of type 1 fibers. $\mathbf{H}$ and $\mathbf{I}$ neurogenic changes with atrophic muscle fascicles and angulated fibers next to normally appearing muscle fibers in HE and Gömori-trichrome staining. I. J Summary of so far published and here described disease-causing variants in SPTBN4. NB, the multi-exon deletion is not marked on the graph. directed against myosin heavy chain slow (MHC neonatal, NCL-MHCn, Novocastra, 1:20) revealed a reduction of type 1 fiber diameters but no manifest fiber-type disproportion (Fig. 2A, B). Both fiber types were nearly equally abundant in age-matched controls of the vastus lateralis muscles. Consistent with these findings, morphometric analysis of muscle fibers from Patient 3 showed that type 1 fiber diameter reduction was higher than in type 2 fibers, (Feret diameter: type $1=21.0 \pm 3.8 \mu \mathrm{m}$ versus type $2=25.6 \pm 4.9 \mu \mathrm{m}$ ), a finding that we know from patients with congenital myopathies, whereas in control muscle (Feret diameter: type $1=26.3 \pm 4.5 \mu \mathrm{m}$ versus type $2=$ $28.3 \pm 5.0 \mu \mathrm{m})$ the difference was significantly smaller. There were no signs of increased fiber diameter variability. Signs of denervation, such as atrophic angulated fibers or fiber grouping, were not observed (Fig. 2G).

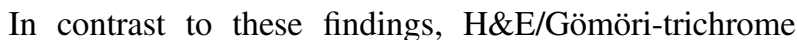
staining of a muscle biopsy sample from Patient 5 showed clear signs of neurogenic changes with atrophic fascicles, dark fibers (type 1) as well as brighter fibers (type 2) small and angulated fibers next to populations of muscle fibers with preserved diameter and groups of small fibers (Fig. $2 \mathrm{H}$, I). ATPase activity at $\mathrm{pH} 9.4$ showed normal fiber differentiation, atrophic fibers were of type 1 and 2 with a similar proportion of both types and groups of both types (not shown).

For IHC we had cryopreserved muscle tissue only from Patient 3 at our disposal. IHC with an antibody directed against $\beta$-spectrin (NCL SPEC1, clone RB C2/3D5, Novocastra UK; 1:100) showed a strong sarcolemmal demarcation of muscle fibers in Patient 3 and an agematched control (Fig. 2C, D). In contrast, IHC with an antibody directed against the non-erythrocytic $\beta I V$-spectrin subtype 4 (sc-368195, H-85, Santa-Cruz, 1:100) showed no staining in Patient 3, while staining was preserved in agematched control muscle (Fig. 2E, F). 


\section{Discussion}

Spectrins are molecular scaffold proteins that link the plasma membrane to the actin cytoskeleton. They are crucial for the determination of cell shape, arrangement of transmembrane proteins, and organelle organization. Variants in SPTBN4 result in ion-channel dysfunction by disrupting the cytoskeletal machinery that controls proper localization of channels and the function of axonal domains in the AIS and in the nodes of Ranvier, where axonal ion channels are normally clustered causing a variety of nervous system dysfunctions.

We have identified multiple novel variants that affect function of SPTBN4 in patients with severe muscular hypotonia, dysphagia, absent speech, delayed gross motor development, and intellectual disability. These symptoms are considered key features of the SPTBN4-related disorder. We have broadened the disorder's clinical spectrum by describing the variably present features of areflexia, axonal motor neuropathy, nystagmus, epileptiform activity in EEG without clinical correlation, and a movement disorders with choreoathetosis.

Analysis of muscle biopsy specimens from affected infants revealed signs of a primary myopathy as well as of secondary neuropathic features. Electrophysiology revealed signs of obvious neuropathy only in Patient 5. This was in accordance with the neuropathic pattern characterized by neurogenic fiber-type grouping in the histopathological studies for this patient. $\beta I V$-spectrin was absent from the sarcolemma of Patient 3, while ATPase staining showed hypotrophic type 1 fibers, but no clear fiber-type disproportion, a finding more characteristic for congenital myopathies. We hypothesize that $\beta \mathrm{IV}$-spectrin deficiency directly impacts the structural stability of the sarcolemma and the initiation or propagation of the depolarization waves along the myofiber and its T-tubular system. We derived that the evidence for a myopathy is mostly from the clinical and histopatholocigal findings, but not from functional studies about the role of SPTBN4 in muscle cells. Further studies are thus needed to determine the impact of pathogenic SPTBN4 variants on the muscle cells proper. Our clinical phenotyping and neurophysiological studies suggest that the muscle weakness seen in patients with SPTBN4 disorder may be caused by a combination of axonal neuropathy and congenital myopathy. This does not rule out the possibility that one pathological principle may dominate as described by Wang et al. (2018), where neuropathy seemed to be the predominant feature.

Though two of our patients did not exhibit clinically manifest seizures, their EEGs showed highly pathologic epileptiform discharges. As $\beta \mathrm{IV}$-spectrin plays a role in the clustering of $K C N Q 2$ subunit-containing potassium channels, there could be some degree of overlap of clinical symptoms with early-infantile epileptic encephalopathy type 7 and with benign familial neonatal seizures type 1 . This is supported by Wang et al. who reported that three of their six patients had clinically manifest epilepsy, with two patients being refractory to antiepileptic medication. As variants in $K C N Q 2$ cause a wide range of phenotypes even within single families with members sharing the same variant [9], we can extrapolate that this may also apply to a protein involved in KCNQ2 clustering. A review of all reported cases of SPTBN4-related disorders shows that epileptic activity and seizures are more common than initially thought. In contrast to the pair of siblings described by Häusler et al. [6], all our patients with a molecular diagnosis of SPTBN4 disorder had severe intellectual disability, indicating that intellectual disability is frequent in this condition. This information must be taken into account when counseling patients and families with SPTBN4 disorder.

We identified and described five additional cases with pathogenic bi-allelic SPTBN4 variants. Four of these were novel and two resulted in a frameshift. The pathogenic homozygous missense variant identified in Patient 3 led the exchange of an evolutionary conserved proline for an arginine. This variant was absent in the gnomAD database and in ClinVar [10]. Patient 5 harbors a multi-exon deletion on the maternally inherited allele and a small insertion leading to a frameshift on the paternally inherited allele. This is the first description of a multi-exon deletion in the SPTBN4 gene and it shows that larger SPTBN4 deletions may account for a part of SPTBN4-related disorder. Screening for large genomic SPTBN4 rearrangements should improve molecular diagnostic rates for this population, in particular for patients where only a single variant that affects function has been identified.

To date, including our report, 15 pathogenic variantstruncating $(n=10)$, missense $(n=4)$, splice-site $(n=1)$ variants, and multi-exon deletion $(n=1)$-have been reported in SPTBN4 [4-6, 11-13] (Fig. 2J). Most affected individuals reported to date are homozygous. In this report we present the first individual with a multi-exon deletion. Patients generally suffer from severe developmental delay and intellectual disability, although two individuals in one family had a milder phenotype, including one individual with normal cognitive development. Speech and language skills are often severely limited. Affected individuals rarely achieve head control and are unable to sit, stand, or walk. They typically have congenital muscular hypotonia. Axonal motor neuropathy leads to hyporeflexia/areflexia and weakness. Most affected individuals require tube feeding. More than half of them develop seizures or have a pathological EEG. The mutations are dispersed over the whole gene and we do not see a clear genotype-phenotype correlation. 
Our study further broadens the clinical and variant spectrum of congenital and early-onset SPTBN4-related disorder. With the accumulation of data on $\beta \mathrm{IV}$-spectrinopathies, it seems rational that SPTBN4 genetic testing should also be considered in patients with early-onset hypotonia, motor developmental delay, and intellectual disability, especially in the presence of axonal neuropathy, deafness, or pathological discharge patterns on the EEG.

Acknowledgements The authors would like to thank the patients and their parents for participation in this study. We would like to thank N. Christine Øien, MS CGC for help with revising the manuscript.

Author contributions All authors read the final version of the manuscript for intellectual content and gave their permission for publication.

Funding This research was funded by grants of the Deutsche Forschungsgemeinschaft (DFG; German Research Foundation) under Germany's Excellence Strategy-EXC-2049-390688087 to MS. Open Access funding enabled and organized by Projekt DEAL.

\section{Compliance with ethical standards}

Conflict of interest The authors declare no competing interests.

Publisher's note Springer Nature remains neutral with regard to jurisdictional claims in published maps and institutional affiliations.

Open Access This article is licensed under a Creative Commons Attribution 4.0 International License, which permits use, sharing, adaptation, distribution and reproduction in any medium or format, as long as you give appropriate credit to the original author(s) and the source, provide a link to the Creative Commons license, and indicate if changes were made. The images or other third party material in this article are included in the article's Creative Commons license, unless indicated otherwise in a credit line to the material. If material is not included in the article's Creative Commons license and your intended use is not permitted by statutory regulation or exceeds the permitted use, you will need to obtain permission directly from the copyright holder. To view a copy of this license, visit http://creativecommons. org/licenses/by/4.0/.

\section{References}

1. Goodman S, Shiffer K. The spectrin membrane skeleton of normal and abnormal human erythrocytes: a review. Am J Physiol-Cell Physiol. 1983;244:C121-41.
2. Berghs S, Aggujaro D, Dirkx R, Maksimova E, Stabach P, Hermel $\mathrm{JM}$, et al. $\beta$ iv spectrin, a new spectrin localized at axon initial segments and nodes of Ranvier in the central and peripheral nervous system. J Cell Biol. 2000;151:985-1002.

3. Komada M, Soriano P. $\beta I V$-spectrin regulates sodium channel clustering through ankyrin-G at axon initial segments and nodes of Ranvier. J Cell Biol. 2002;156:337-48.

4. Knierim E, Gill E, Seifert F, Morales-Gonzalez S, Unudurthi SD, Hund TJ, et al. A recessive mutation in beta-IV-spectrin (SPTBN4) associates with congenital myopathy, neuropathy, and central deafness. Hum Genet. 2017;136:903-10.

5. Wang C-C, Ortiz-González XR, Yum SW, Gill SM, White A, Kelter E, et al. $\beta I V$ spectrinopathies cause profound intellectual disability, congenital hypotonia, and motor axonal neuropathy. Am J Hum Genet. 2018. https://doi.org/10.1016/j.ajhg.2018.04. 012

6. Häusler MG, Begemann M, Lidov HG, Kurth I, Darras BT, Elbracht M. A novel homozygous splice-site mutation in the SPTBN4 gene causes axonal neuropathy without intellectual disability. Eur J Med Genet. 2020;63:103826.

7. Derks MFL, Harlizius B, Lopes MS, Greijdanus-van der Putten SWM, Dibbits B, Laport K. et al. Detection of a frameshift deletion in the SPTBN4 gene leads to prevention of severe myopathy and postnatal mortality in pigs. Front Genet. 2019; 10:1226

8. Richards S, Aziz N, Bale S, Bick D, Das S, Gastier-Foster J, et al. Standards and guidelines for the interpretation of sequence variants: a joint consensus recommendation of the American College of Medical Genetics and Genomics and the Association for Molecular Pathology. Genet Med. 2015;17:405-23.

9. Borgatti R, Zucca C, Cavallini A, Ferrario M, Panzeri C, Castaldo $\mathrm{P}$, et al. A novel mutation in KCNQ2 associated with BFNC, drug resistant epilepsy, and mental retardation. Neurology. 2004;63: $57-65$.

10. Karczewski KJ, Francioli LC, Tiao G, Cummings BB, Alföldi J, Wang $\mathrm{Q}$, et al. The mutational constraint spectrum quantified from variation in 141,456 humans. Nature. 2020;581:434-43.

11. Anazi S, Maddirevula S, Salpietro V, Asi YT, Alsahli S, Alhashem A, et al. Expanding the genetic heterogeneity of intellectual disability. Hum Genet. 2017;136:1419-29.

12. Monies D, Abouelhoda M, Assoum M, Moghrabi N, Rafiullah R, Almontashiri $\mathrm{N}$, et al. Lessons learned from large-scale, first-tier clinical exome sequencing in a highly consanguineous population. Am J Hum Genet. 2019;104:1182-201.

13. Pehlivan D, Bayram Y, Gunes N, Coban Akdemir Z, Shukla A, Bierhals $\mathrm{T}$, et al. The genomics of arthrogryposis, a complex trait: candidate genes and further evidence for oligogenic inheritance. Am J Hum Genet. 2019;105:132-50.

14. Köhler S, Carmody L, Vasilevsky N, Jacobsen JOB, Danis D, Gourdine JP, et al. Expansion of the Human Phenotype Ontology (HPO) knowledge base and resources. Nucleic Acids Res. 2018;47:D1018-27. 